\title{
The clinical analysis of acute pancreatitis in colorectal cancer patients undergoing chemotherapy after operation
}

This article was published in the following Dove Press journal:

OncoTargets and Therapy

II September 2015

Number of times this article has been viewed

\author{
Yanlei Ji' \\ Zhen $\mathrm{Han}^{2}$ \\ Limei Shao' \\ Yunling Li' \\ Long Zhao' \\ Yuehuan Zhao' \\ 'Department of Special Diagnosis, \\ Shandong Cancer Hospital and \\ Institute, Jinan, People's Republic \\ of China; ${ }^{2}$ Department of Internal \\ Medicine, Jinan Second People's \\ Hospital, Jinan, People's Republic \\ of China
}

Correspondence: Yuehuan Zhao Department of Special Diagnosis, Shandong Cancer Hospital and Institute, 440 Jiyan Road, Jinan 250 I 17, Shandong Province, People's Republic of China

Tel +8653I 67626239

Fax +86 531 67626239

Email yuehuanxczhao@।63.com
Abstract: Acute pancreatitis is a rare complication in postoperative colorectal cancer patients after FOLFOX6 (oxaliplatin + calcium folinate +5-FU [5-fluorouracil]) chemotherapy. In this paper, a total of 62 patients with gastrointestinal cancer were observed after the burst of acute pancreatitis. Surgery of the 62 cases of colorectal cancer patients was completed successfully. But when they underwent FOLFOX6 chemotherapy, five patients got acute pancreatitis $(8.06 \%)$, four $(6.45 \%)$ had mild acute pancreatitis, and one $(1.61 \%)$ had severe acute pancreatitis, of which two were males $(3.23 \%)$ and three females $(4.84 \%)$. No patients $(0.00 \%)$ had acute pancreatitis on the 1 st day after chemotherapy; one patient (1.61\%) got it in the first 2 and 3 days after chemotherapy; and three others $(4.83 \%)$ got it in the first 4 days after chemotherapy. In the 62 patients with malignant tumors, the body mass index (BMI) was less than 18 (underweight) in six of them, with two cases of acute pancreatitis (33.33\%); the BMI was 18-25 (normal weight) in 34 cases, with one case $(2.94 \%)$ of acute pancreatitis; the BMI was $25-30$ (overweight) in 13 cases, with 0 cases $(0.00 \%)$ of acute pancreatitis; and the BMI was $\geq 30$ (obese) in nine patients, with two cases of acute pancreatitis (22.22\%). After symptomatic treatment, four patients were cured and one died; the mortality rate was $1.61 \%$. Most of them appeared in the first 4 days after chemotherapy; the probability of this complication is significantly higher in slim and obese patients than in normal weight patients. Postoperative colorectal cancer patients after FOLFOX6 chemotherapy have a sudden onset of acute pancreatitis occult, especially in patients with severe acute pancreatitis; the symptoms are difficult to control, there is high mortality and it is worthy of clinician's attention.

Keywords: colorectal cancer, postoperative, acute pancreatitis, chemotherapy

\section{Introduction}

The incidence and mortality of colorectal cancer in our country have gradually increased in recent years, and it has risen to one among the first four malignant tumors. At present, a comprehensive treatment consisting of surgical treatment and supplemented chemotherapy has been widely applied clinically, and adaptability for the colorectal cancer patients is also required. ${ }^{1}$

Oxaliplatin, a third-generation platinum compound alkylating agent with the function of inhibiting DNA replication, is effective against advanced colorectal cancer by combination action with folinic acid and 5-fluorouracil (5-FU) or capecitabine. This combined method is called FOLFOX6 and XELOX regimens..$^{2-4}$ In 1999, the FOLFOX6 (oxaliplatin + leucovorin +5 -FU) chemotherapy program was first confirmed to have good results for the second-line treatment of advanced colorectal cancer, which has been gradually applied in the domestic medical fields. ${ }^{5-7}$ At the target point, Oxaliplatin could easily covalently combine with the G DNA chain and may form intrachain 
crosslinking, chain crosslinking, and DNA-protein crosslinkings, leading to DNA damage and destruction of DNA replication and finally killing cancer cell via toxic effect. ${ }^{8-10}$ Oxaliplatin has almost no side effects, with the exception of peripheral neuropathy. ${ }^{11}$ Lee et $\mathrm{l}^{12}$ reported that the important grade 3-4 toxicities were neutropenia (34.1\%), leukopenia (7.3\%), nausea (9.8\%), and vomiting (7.3\%). However, chemotherapy drugs cannot completely avoid damage to normal tissue, ${ }^{13,14}$ especially the damage to the digestive organs, which is one of the main obstacles in improving the effect of chemotherapy. As pancreatic injury (especially for acute pancreatitis) and the related complications may lead to death, the timely detection, treatment, and prevention of serious adverse reactions are an important guarantee to complete the chemotherapy plan. However, up to now there is little domestic and foreign contribution devoted to the sudden pancreatitis after chemotherapy at home and abroad. Therefore, currently, a systematic research work on the colorectal cancer patients undergoing chemotherapy after operation is very important to choose the reasonable chemotherapy regimens to reduce the adverse reactions caused by chemotherapy drugs and to improve the survival rate of patients.

In this paper, to show the incidence of acute pancreatitis in the patients postchemotherapy, the acute pancreatitis cases in postoperative colorectal cancer patients, in our department, caused by chemotherapy with FOLFOX6 are analyzed and reported.

\section{Materials and methods Clinical data}

This study was approved by the Ethics Committee of Shandong Cancer Hospital and Institute, and written informed consent was obtained from the patients. Sixty-two patients with colorectal cancer from Shandong Cancer Hospital were admitted from February 2012 to February 2013, including 39 males and 23 females, aged from 36 to 67 years, with a mean age of $49 \pm 6.9$ years. All patients were confirmed for Phase II or III colorectal adenocarcinoma after radical operation, including 39 cases of colon adenocarcinoma, 23 cases of rectal adenocarcinoma, 20 cases of high differentiation adenocarcinoma, 31 cases of moderately differentiated adenocarcinoma, and eleven cases of poorly differentiated adenocarcinoma. Inclusion criteria were as follows: 1) those who underwent resection of colorectal cancer, postoperative specimens were diagnosed as stage II or III colorectal cancer by pathology or cytology; 2) preoperative imaging studies had found no basis disorders for the pancreas and biliary system; 3) without preoperative chemotherapy or those who had not received other anticancer therapy for 6 months; 4) normal bone marrow function $\left(\mathrm{WBC}>4.0 \times 10^{9} / \mathrm{L}\right.$, platelets $>100 \times 10^{9} / \mathrm{L}$, hemoglobin $>90 \mathrm{~g} / \mathrm{L}$ ); and 5) liver and kidney function was normal, and no heart disease, diabetes, and other underlying diseases. Patients with previous alcohol consumption and biliary stones diseases should be excluded. All patients were treated with regimens of FOLFOX6 and had signed informed consent before treatment.

5 -FU was injected at $400 \mathrm{mg} / \mathrm{m}^{2}$ on day one, then $2,400 \mathrm{mg} / \mathrm{m}^{2}$ continuous intravenous infusion for 46 hours; $100 \mathrm{mg} / \mathrm{m}^{2}$ oxaliplatin was given on day one; leucovorin at $400 \mathrm{mg} / \mathrm{m}^{2}$ for 2 hours, on day one. The treatment for one period was 21 days, and the patients received a total of four to six periods of treatment. After treatment, judgment of the curative effect and side reaction of record, deep vein intubation, or central venous catheter to prevent the occurrence of phlebitis, avoiding cold stimulation during the use of Oxaliplatin, paying attention to keep warm, taking antiemetic, gastric mucosal protective drugs before and after chemotherapy, as well as review of blood count, liver function, renal function, blood, and urine amylase. The leukogenic drugs, such as granulocyte colony-stimulating factor, should be initiated when neutrophils $<115 \times 10^{9} / \mathrm{L}$ or WBC $<2.0 \times 10^{9} / \mathrm{L}$.

High-calorie, high-protein, high-vitamin, and easily digestible liquid or semiliquid diet should be given during chemotherapy, avoiding foods that are overheating, supercooling or excitant, lest they cause gastrointestinal discomfort. Enteral and parenteral nutritional supplements as well as intravenous supplement are two main ways of providing nutritional supplements. As the basic need of heat capacity of every human body is $104.6 \mathrm{~kJ} / \mathrm{kg}$, the necessary amounts intravenous fat emulsion, amino acids, glucose and saline, albumin, and appropriate albumin were given to patients.

\section{The diagnostic criteria for acute pancreatitis}

Currently, there is no uniform standard for the diagnosis of acute pancreatitis in our country. The Chinese Medical Association formulated the standard of diagnosis and treatment of acute pancreatitis in the People's Republic of China Guide in 2013. The standard is as follows: clinically, if there were two of the following three characteristics, it would be diagnosed as acute pancreatitis: 1) in accordance with acute pancreatitis, acute abdominal pain, sudden, persistent, severe abdominal pain, sometimes radiating to the back; 2) serum amylase and (or) lipase activity at least three times higher than the upper limit of normal value; and 3) enhanced CT/ MRI or abdominal ultrasound show images in patients with sudden pancreatitis after chemotherapy (Figure 1). 

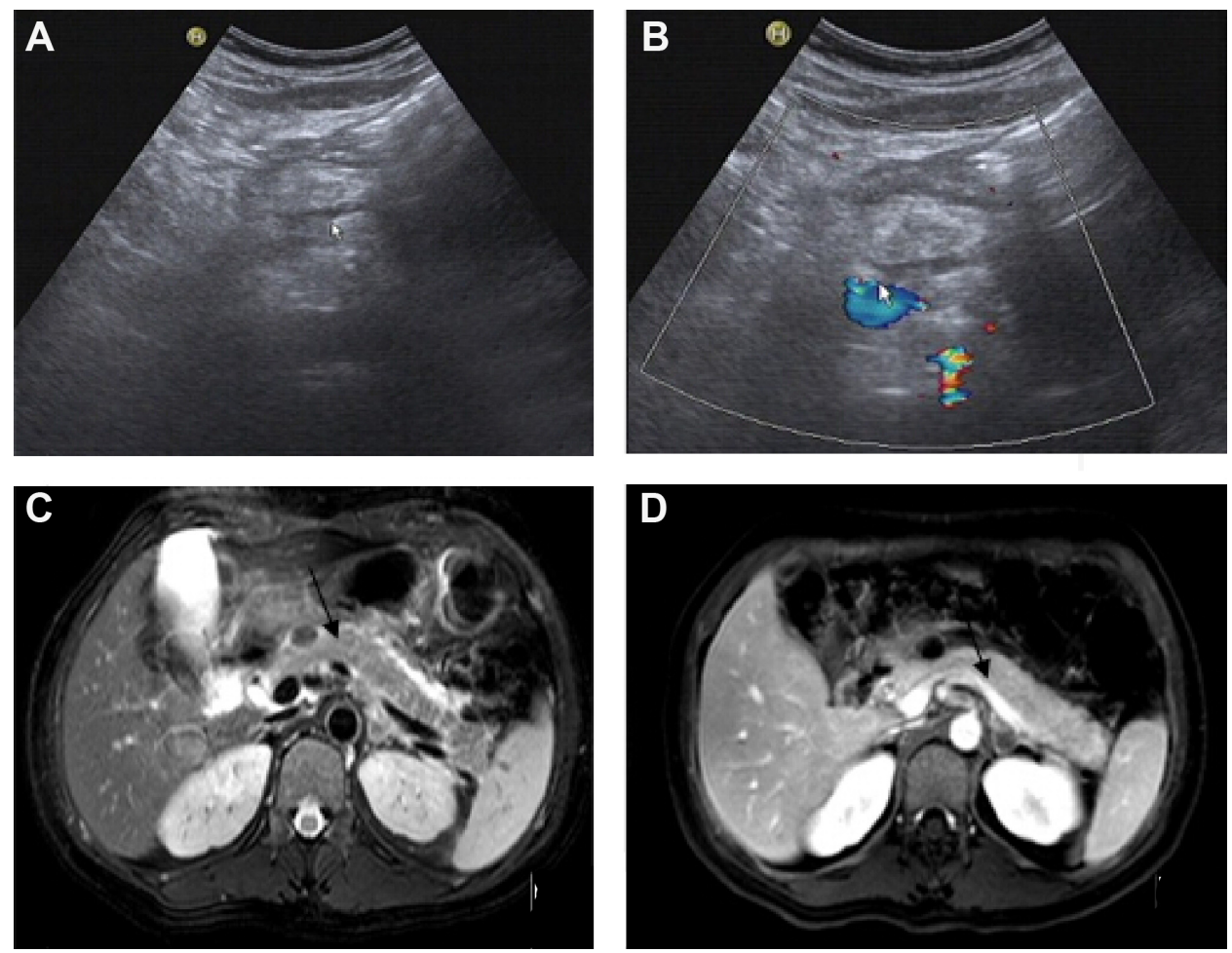

Figure I (A, B) B-Ultrasound images in patients with sudden pancreatitis after chemotherapy. The small white arrows indicate the pancreatic necrotic tissues. (C, D) Magnetic resonance images in patients with sudden pancreatitis after chemotherapy.

\section{Statistical methods}

All statistical analyses were calculated using the SPSS software (version 13.0 J, SPSS Inc., Chicago, IL, USA), and $P$-value $<0.05$ was considered statistically significant. Count data were compared using $\chi^{2}$-test, whereas continuous variables that were measured by the mean \pm standard deviation $(x \pm s)$ were compared using the Student's $t$-test.

\section{Results}

Patients with acute pancreatitis cases and the proportion of the light and severe

\section{pancreatitis}

Sixty-two colorectal cancer patients who successfully completed surgery underwent FOLFOX6 chemotherapy, of which five patients had acute pancreatitis $(8.06 \%)$ during chemotherapy, four patients $(6.45 \%)$ had mild acute pancreatitis, and one case $(1.61 \%)$ was severe acute pancreatitis (Table 1).

\section{Differences between the proportion of men and women with postoperative chemotherapy burst pancreatitis}

Of the five patients with acute pancreatitis, two were males, with an incidence rate of $3.23 \%$, and three were females, with an incidence rate of $4.84 \%$. There was no significant difference between men and women $\left(\chi^{2}=0.39, P>0.05\right)$.

\section{Relationship between body mass index and the burst of pancreatitis after chemotherapy with FOLFOX6}

Of the 62 patients with malignant tumors, the body mass index (BMI) was $\leq 18$ (underweight) in six patients, and there were two cases of acute pancreatitis with a rate of $33.33 \%$; the BMI was 18-25 (normal weight) in 34 patients, and there was one case of acute pancreatitis with a rate of $2.94 \%$; and the BMI was $25-30$ (overweight) in 13 patients, and there was 0 cases of acute pancreatitis with a rate of

Table I In the study group comparing individual indicators

\begin{tabular}{|c|c|c|c|c|}
\hline & Onset (n) & Without disease (n) & $\chi^{2}$ & $P$-value \\
\hline \multicolumn{5}{|c|}{ BMI $\left(\mathrm{kg} / \mathrm{m}^{2}\right)$} \\
\hline $18-25$ & I & 33 & & \\
\hline$\leq 18$ & 2 & 4 & 6.790 & $0.009^{a}$ \\
\hline$\geq 30$ & 2 & 7 & 4.076 & $0.043^{b}$ \\
\hline \multicolumn{5}{|c|}{ Myelosuppression } \\
\hline Yes & 4 & 10 & 10.257 & 0.001 \\
\hline No & 1 & 47 & & \\
\hline \multicolumn{5}{|l|}{ Sex } \\
\hline Female & 2 & 37 & 0.388 & $>0.05$ \\
\hline Male & 3 & 20 & & \\
\hline
\end{tabular}

Notes: ${ }^{a}$ The comparison of the incidence of acute pancreatitis between patients with medium build $(\mathrm{BMI}=\mid \mathrm{8}-25)$ and emaciated patients $(\mathrm{BMI}<\mathrm{I} 8)$ who underwent chemotherapy after operation. ${ }^{\mathrm{b}}$ The comparison of the incidence of acute pancreatitis between patients with medium build $(B M I=\mid 8-25)$ and corpulent patients $(B M I>30)$ who underwent chemotherapy after operation.

Abbreviation: BMI, body mass index. 
$0.00 \%$. The BMI was $\geq 30$ (obese) in nine patients, and there were two cases of acute pancreatitis with a rate of $22.22 \%$. The incidence of acute pancreatitis with chemotherapy after operation for medium stature patients (BMI $=18-25)$ was significantly lower than that in lean patients $(\mathrm{BMI}<18)$ $\left(\chi^{2}=6.79, P<0.05\right)$ and that in obese patients $(\mathrm{BMI}>30)$ $\left(\chi^{2}=4.076, P<0.05\right)$.

\section{The duration of sudden onset of acute pancreatitis from the start of chemotherapy}

No case got acute pancreatitis on the 1 st day after chemotherapy, with the rate of $0.00 \%$; one case got acute pancreatitis in the first 2 days after chemotherapy, with the rate of $1.61 \%$; one case got acute pancreatitis in the first 3 days after chemotherapy, with the rate of $1.61 \%$; and three cases got acute pancreatitis in the first 4 days after chemotherapy, with the rate of $4.83 \%$. Obviously, acute pancreatitis often occurred in the first 4 days after chemotherapy. One case in the study had died of acute hemorrhagic necrotizing pancreatitis in the first 4 days after the start of chemotherapy and this may be related to the chemotherapy drug accumulation and the person being obese.

\section{Relationship between bone marrow suppression and acute pancreatitis}

Of the 62 patients after chemotherapy, 14 had bone marrow suppression after chemotherapy, and four of the five cases of acute pancreatitis patients also had bone marrow suppression after chemotherapy, in which one case was complicated with IV arrest of bone marrow (according to the standard chemotherapy toxicity assessment of WHO, the bone marrow suppression is divided into $0-\mathrm{IV}$ ). The rate of pancreatitis patients with concomitant bone marrow suppression was $80.00 \%$; ten cases in 57 patients who did not have acute pancreatitis after chemotherapy had bone marrow suppression; the incidence rate was $17.54 \%$, which was significantly different from the incidence $\left(\chi^{2}=10.26, P<0.05\right)$.

\section{The onset symptoms of acute pancreatitis and the symptoms prognosis of patients after symptomatic treatment}

In five patients with acute pancreatitis, the first symptom was pain in the upper abdominal region, accompanied by nausea and vomiting. Four patients were cured after active symptomatic treatment, whereas one died because of acute hemorrhagic necrotizing pancreatitis accompanied with severe bone marrow suppression; the mortality was $20 \%$.

\section{Discussion}

According to relevant statistics, among all types of cancers, colorectal cancer is the fourth most common morbidity causing malignant tumor in the world. With the development of our society, and the change in our eating habits, there are more and more people suffering with this kind of disease. Because of the insidious onset of colorectal cancer, it is always difficult to notice it in the early stage. By the time the clinical symptoms of colorectal cancer appear, such as intestinal obstruction, changes in the stool, weight loss, and other physical symptoms, it has developed to the advanced stage. As a result, nearly one-third of the patients lose the chance of surgical treatment, accompanied with the large possibility of recurrence or metastasis in 5 years. Therefore, clinically, comprehensive treatment as in operation combined with chemotherapy has been widely applied for colorectal cancer patients. ${ }^{15}$

On the basis of different chemotherapy drugs, various regimens have been developed. FOLFOX6 is one of the most common programs for colorectal cancer treatment, which includes 5-FU, oxaliplatin, and leucovorin. The efficiency rate of 5-FU for colorectal cancer is approximately 30\%, and it has been the most widely used chemotherapy drug in colorectal cancer, whose pharmacologic action is the inhibition of DNA synthesis for cancer cell. ${ }^{16-18} 5-\mathrm{FU}$ is a cell cycle-specific drug, with killing effect on proliferation of the cells. However, the effect of simple application of the drug is not ideal, because its initial treatment efficiency is approximately $30 \%$ and retreatment efficiency is approximately $20 \%$. Therefore, it should be applied in combination with other drugs.

Following cisplatin and carboplatin, oxaliplatin is the third-generation platinum anticancer drug, which could be covalently cross-linked to $\mathrm{G}$ (guanine) on the DNA chain. The tumor cell DNA replication could be destroyed by chain cross-linking between chains. ${ }^{8-10}$ According to clinical studies, ${ }^{8,9}$ oxaliplatin obviously inhibits the growth of colorectal cancer cell, generating synergistic effect when combined with 5-FU. However, chemotherapy drugs cannot completely avoid the damage of normal tissues, especially those of the digestive organs, which are the main obstacles to raising the therapeutic effect. As a result, pancreatic injury (mainly, acute pancreatitis) becomes the most common morbidity complications in colorectal cancer.

In the group trial, 62 colorectal cancer patients successfully completed their surgery, following FOLFOX6 chemotherapy. During chemotherapy, five patients suffered from acute pancreatitis, and the rate of complication was 
$8.06 \%$, including mild acute pancreatitis in four patients, with a rate of $6.45 \%$, and acute necrotizing pancreatitis in one patient, with the rate of $1.61 \%$. Of all five cases of acute pancreatitis, two were males, with a morbidity $3.23 \%$, and three were females, with a morbidity $4.84 \%$, the difference in morbidity between men and women was not significant $\left(\chi^{2}=0.39, P>0.05\right)$. The symptoms of pancreatitis induced by chemotherapy are usually mild, but very few patients may develop acute hemorrhagic necrotizing pancreatitis because of congenital biliary tract disease, BMI, and severe bone marrow suppression. The acute pancreatitis in the study group may be disproportionate because of insufficient samples, and thus, further study is required. There was no significant difference observed between men and women in postoperative complications of acute pancreatitis induced by chemotherapy. Previous studies ${ }^{19,20}$ have shown that men are more prone to acute pancreatitis than women, mainly because of the number of male drinkers who more frequently attend social events and are more likely to overeat and drink heavily. However, the hospitalized patients were not exposed to alcohol or allowed to overeat or drink heavily, and so the two sets of results are not inconsistent. Acute pancreatitis caused as a result of sexual differences among patients who underwent chemotherapy after surgery was not visible.

Of the 62 patients with malignant tumors, the BMI was $\leq 18$ (underweight) in six patients, of whom two got acute pancreatitis with a rate of $33.33 \%$; the BMI was 18-25 (normal weight) in 34 patients, of whom one got acute pancreatitis with a rate of $2.94 \%$; the BMI was 25-30 (overweight) in 13 patients, of whom 0 cases $(0.00 \%)$ got acute pancreatitis; and the BMI was $\geq 30$ (obese) in nine patients, of whom two cases got acute pancreatitis with the rate of $22.22 \%$. As the nutritional status of each patient was different, the body resistance and the sensitivity to chemotherapeutic drugs, and the digestive tract and digestive organs that were affected by the chemotherapeutic drugs were very different. Differences in BMI of patients with bone marrow suppression lead to the different body resistance of bacteria. It is obvious that the low immunity leads to being more vulnerable to bacterial infection and high morbidity of pancreatitis.

Many foreign studies have demonstrated that obese people have high morbidity of acute pancreatitis and severe pancreatitis, ${ }^{19-21}$ and they use BMI as a diagnostic indicator for obesity. Although the BMI was different among patients $\left(>25,>28\right.$, and $>30 \mathrm{~kg} / \mathrm{m}^{2}$ ), the results strongly suggest that the incidence of acute pancreatitis in obese or overweight people was significantly higher than those of normal weight people. The proportion of local or systemic complications, pancreatitis severity, and mortality in the patients who are obese or overweight was higher. ${ }^{22}$ The role of obesity in the pathogenesis of acute pancreatitis has not been fully elucidated. Several possible reasons could be listed as follows: 1) obesity, especially in men, as obesity often means a lot of abdominal and visceral fat accumulation, and excessive deposition of fat around the pancreas may lead to pancreatic hemorrhage, necrosis, as well as saponification reactions; 2) fat decomposition produces large amounts of free fatty acids, directly or indirectly, which can damage pancreatic microcirculation; 3) obesity is often associated with metabolic changes, such as dyslipidemia, insulin resistance, making cholecystitis, cholelithiasis, coronary artery disease, hypertension, high cholesterol, etc, which increases the incidence of chronic diseases. Meanwhile, their obese state is related to chronic inflammation of the body, which increases sensitivity to proinflammatory factors of the pancreas; and 4) more fat tissue necrosis accumulates around the pancreatic in obese patients under acute pancreatitis and will result in a large number of toxic substances, increasing pancreatic necrosis. These toxic substances can loop through the liver and kidneys. Adipose tissue is not merely a passive participant in the form of energy storage, but also an endocrine organ, secreting cytokines such as TNF- $\alpha$, IL-6, IL-8, leptin, adiponectin, resistin, etc, which increase inflammation. Leptin plays a role in the body's immune system, and it can regulate the function of T-lymphocytes involved in the regulation of the systemic inflammatory response..$^{23-25}$ In short, obesity may be associated with high triglyceride hyperlipidemia synergies, making acute pancreatitis even worse, and possibly associating with a number of chronic diseases, which indirectly affect the severity of acute pancreatitis, whose exact mechanism needs further study.

In our work, no patient got acute pancreatitis on the $1 \mathrm{st}$ day after chemotherapy, with a rate of $0.00 \%$; one patient got acute pancreatitis in the first 2 days after chemotherapy, with a rate of $1.61 \%$; one got acute pancreatitis in the first 3 days after chemotherapy, with a rate of $1.61 \%$; and three got acute pancreatitis in the first 4 days after chemotherapy, with a rate of $4.83 \%$. The study found that $60 \%$ of patients got acute pancreatitis on the 2 nd day after chemotherapy, and one case of acute necrotizing pancreatitis died on the 2nd day after chemotherapy, with the sudden appearance of refractory shock and severe bone marrow suppression. Hence, it is of great risk for patients receiving chemotherapy to be immediately discharged at the end of chemotherapy. At the end of chemotherapy, patients should continue to 
be hospitalized for 1-2 days to be under observation and to have a better review of blood routine, blood amylase, and urine amylase to discover hidden acute pancreatitis, to enable symptomatic treatment and to avoid hemorrhagic necrotizing pancreatitis before being discharged. We have to be vigilant and check for acute pancreatitis when patients have abdominal pain, nausea, vomiting, and other symptoms during chemotherapy. Moreover, during the period of chemotherapy, patients have poor appetite due to adverse reactions of drugs in the gastrointestinal tract, while the day after chemotherapy, their appetite improve significantly and they begin overeating, which in turn increases the probability of occurrence of pancreatitis.

The toxic side effects of cytotoxic drugs on the bone marrow suppression caused by bone marrow hematopoietic stem cells are the most common and most serious acute adverse reactions, mainly manifested as neutropenia. Leukocytes play an important part in recovery from injury in the body, in resisting pathogen invasion, and in providing immunity. They are important inflammatory cells in the body that have phagocytosis and the control of cell movement. The inflammatory reaction is a double-edged sword to the body, and neutrophils plays very important role in anti-inflammatory and immunomodulation. ${ }^{26,27}$ If the number of white blood cells is too low, the body will be subjected to significantly higher chance of infection. Some anti-inflammatory and proinflammatory cytokines with bacteria and their metabolites usually tend to have some degree of pancreatic damage, and accordingly, the incidence of acute pancreatitis increases. In the study group, one patient with acute hemorrhagic necrotizing pancreatitis had white blood cell count almost close to zero and the body skin was scattered with foci of infection.

The main side effects of chemotherapy are acute toxicity, of which acute pancreatitis is relatively rare. The reasons for the pancreatitis may be related to being lean or obese, the low immune ability of the body, the chemotherapy causing hyperleukocytosis, the disorders of digestion, the bile reflux caused by sicchasia activated pancreatin, and pancreatitis induced by other reasons. As there was one patient who suddenly presented with acute hemorrhagic necrotic pancreatitis and died after chemotherapy, we need to treat patients with adverse reactions of chemotherapy in a timely manner. In case of sudden rapid shallow breathing, oliguria, shock, the peritoneal irritation sign, and any other clinical manifestations with the primary disease that cannot be explained, there should be particular vigilance in case severe acute pancreatitis develops.
In recent years, with improved living conditions, protein and fat intake, decreased physical activity, increased blood pressure, and a series of other factors, the number of obese population rises year by year. At the same time, due to the surge in the incidence of tumors attributed to environmental pollution, chemotherapy has been widely used in clinical oncology, and chemotherapy drug-induced pancreatitis has also increased accordingly in proportion to patients. Clinicians should improve the understanding of adverse drug reactions of chemotherapy (especially in patients with gastrointestinal cancer undergoing FOLFOX chemotherapy), such as sudden severe upper abdominal pain with nausea and vomiting, in addition to considering chemotherapy-induced gastrointestinal reactions. They also need to guard against the occurrence of acute pancreatitis, and closely monitor trypsin and other indicators, through laboratory tests or radiologic confirmation, and should promptly disable chemotherapy drugs in order to avoid serious consequences according to the principles of treatment of acute pancreatitis.

\section{Conclusion}

In summary, although FOLFOX6 programs have certain poisonous side effects, most patients can be safely and steadily treated through the period of chemotherapy by timely investigation of chemotherapy complications and appropriate treatment measures. As the acute pancreatitis induced by chemotherapy were usually mild, and FOLFOX6 regimen has the exact curative effect on advanced colorectal cancer patients, it is worth recommending chemotherapy to improve survival time and quality of life of patients.

\section{Acknowledgment}

There is no funding supported to this paper.

\section{Disclosure}

The authors report no conflicts of interest in this work.

\section{References}

1. Grosso F, Mandala M, Maglione V, et al. Neoadjuvant therapy and mini-invasive total mesorectal excision for rectal cancer: feasibility and outcome analysis from a single institution prospectively collected data base. Tumori. 2012;98:689-695.

2. Maindrault-Goebel F, Louvet C, André T, et al. Oxaliplatin added to the simplified bimonthly leucovorin and 5 -fluorouracil regimen as second-line therapy formetastatic colorectal cancer (FOLFOX6). Eur J Cancer. 1999; $35: 1338-1342$.

3. Twelves C. Can capecitabine replace 5-FU/leucovorin in combination with oxaliplatin for the treatment of advanced colorectal cancer? Oncology. 2002;16:23-26.

4. De Gramont A, Figer A, Seymour M, et al. Leucovorin and fluorouracil with or without oxaliplatin as first-line treatment in advanced colorectal cancer. J Clin Oncol. 2000;18:2938-2947. 
5. Kaneko J, Aoyagi H, Yoshida T, et al. A patient with sigmoid colon cancer (c Stage IV) who responded to preoperative chemotherapy with mFOLFOX6 plus bevacizumab and was subsequently able to undergo a radical operation. Gan To Kagaku Ryoho. 2012;39:2204-2206.

6. Michael M, Zalcberg J, Gibbs P, et al. A phase I trial of imatinib in combination with mFOLFOX6-bevacizumab in patients with advanced colorectal cancer. Cancer Chemother Pharmacol. 2013;71:321-330.

7. Kato K, Inaba Y, Tsuji Y, et al. A multicenter phase-II study of 5-FU, leucovorin and oxaliplatin (FOLFOX6) in patients with pretreated metastatic colorectal cancer. Jpn J Clin Oncol. 2011;41:63-68.

8. Alian OM, Azmi AS, Mohammad RM, et al. Network insights on oxaliplatin anti-cancer mechanisms. Clin Transl Med. 2012;1:26.

9. Shoeib T, Sharp BL. Interactions of oxaliplatin with the cytoplasmic thiol containing ligand glutathione. Metallomics. 2012;4(12):1308-1320.

10. Mowaka S, Ziehe M, Mohamed D, et al. Structures of oxaliplatinoligonucleotide adducts from DNA. J Mass Spectrom. 2012;47: 1282-1293

11. Cassidy J, Misset JL. Oxaliplatin-related side effects: characteristics and management. Semin Oncol. 2002;29(5 Suppl 15):11-20.

12. Lee HH, Hur H, Kim SH, Park AR, Kim W, Jeon HM. Outcomes of modified FOLFOX-6 as first line treatment in patients with advanced gastric cancer in a single institution; retrospective analysis. Cancer Res Treat. 2010;42:18-23.

13. Shioiri M, Hasegawa M, Tanaka H, et al. Two cases of interstitial pneumonia caused by cetuximab plus mFOLFOX6 therapy in metastatic colorectal cancer patients. Gan To Kagaku Ryoho. 2012;39:2198-2200.

14. Manegold C, Gatzemeier U, von Pawel J, et al. A case of 5-fluorouracilinduced hyperammmonia after chemotherapy for metastatic colon cancer. Nihon Shokakibyo Gakkai Zasshi. 2009;106:1744-1750.

15. Gill S, Goldberg RM. First-line treatment strategies to improve survival in patients with advanced colorectal cancer. Drugs. 2004;64:27-44.

16. Nishi T, Hamamoto Y, Uemoto J, et al. Modified FOLFOX6 (mFOLFOX6) in metastatic colorectal carcinoma patients with poor performance status. Gan To Kagaku Ryoho. 2012;39:2557-2560.
17. Hoff PM, Hochhaus A, Pestalozzi BC, et al. Cediranib plus FOLFOX CAPOX versus placebo plus FOLFOX/CAPOX in patients with previously untreated metastatic colorectal cancer: a randomized, double-blind, phase III study (HORIZON II). J Clin Oncol. 2012;30:3596-3603.

18. Tsutsumi S, Watanabe R, Tabe Y, et al. Scheduled prospective tri-weekly modified FOLFOX6 maintenance chemotherapy in the treatment of metastatic colorectal cancer. Hepatogastroenterology. 2011;58:1930-1932.

19. Healey E, Stillfried GE, Eckermann S, et al. Comparative effectiveness of 5-fluorouracil with and without oxaliplatin in the treatment of colorectal cancer in clinical practice. Anticancer Res. 2013;33:1053-1060.

20. Chen ML, Fang CH, Liang LS, et al. A meta-analysis of chemotherapy regimen fluorouracil/leucovorin/oxaliplatin compared with fluorouracil/leucovorin in treating advanced colorectal cancer. Surg Oncol. 2010;19:38-45.

21. Petrov MS. Editorial: abdominal fat: a key player in metabolic acute pancreatitis. Am J Gastroenterol. 2013;108:140-142.

22. Sadr-Azodi O, Orsini N, Andren-Sandberg A, et al. Abdominal and total adiposity and the risk of acute pancreatitis: a population-based prospective cohort study. Am J Gastroenterol. 2013;108:133-139.

23. Guzman Calderon E, Montes Teves P, Monge Salgado E, et al. Bisap-O obesity included in score BISAP to improve prediction of severity in acute pancreatitis. Rev Gastroenterol Peru. 2012;32:251-256.

24. Chen SM, Xiong GS, Wu SM. Is obesity an indicator of complications and mortality in acute pancreatitis? An updated meta-analysis. J Dig Dis. 2012;13:244-251.

25. Al-Suhaimi EA, Shehzad A. Leptin, resistin and visfatin: the missing link between endocrine metabolic disorders and immunity. Eur J Med Res. 2013;18:12.

26. Liang C, Liao J, Deng Z, et al. Leptin attenuates lipopolysaccharideinduced apoptosis of thymocytes partially via down-regulation of cPLA2 and p38 MAPK activation. Int Immunopharmacol. 2013;15: 620-627.

27. Behnes M, Brueckmann M, Lang S, et al. Alterations of leptin in the course of inflammation and severe sepsis. BMC Infect Dis. 2012;14:217.
OncoTargets and Therapy

\section{Publish your work in this journal}

OncoTargets and Therapy is an international, peer-reviewed, open access journal focusing on the pathological basis of all cancers, potential targets for therapy and treatment protocols employed to improve the management of cancer patients. The journal also focuses on the impact of management programs and new therapeutic agents and protocols on

\section{Dovepress}

patient perspectives such as quality of life, adherence and satisfaction. The manuscript management system is completely online and includes a very quick and fair peer-review system, which is all easy to use. Visit http://www.dovepress.com/testimonials.php to read real quotes from published authors. 